\title{
Going transparent
}

\author{
Nature Microbiology will offer authors the option of publishing a peer-review file that includes anonymous \\ peer-review reports, author responses and our decision letters. We will also request that articles include more \\ source data and are more transparent in reporting data availability.
}

A new year usually brings new resolutions aimed at improving some aspect of our lives. Likewise, Nature Microbiology has recently introduced two initiatives that will come to light in primary research published in 2020 and reflect our desire to increase the transparency of our peer-review process and the reproducibility of the research we publish.

The authors of primary research articles submitted from 1 December 2019 will have the choice of publishing a peer-review file with their article that includes the referee comments to authors, the author responses and all of our decision letters. This option will be offered at various stages of the process, but authors can delay making a decision until acceptance. On the other hand, by agreeing to referee for Nature Microbiology, reviewers will accept that their anonymous reports may ultimately be published, of which they will be reminded at various times during the process (the referee invitation letter, the e-mail with the link to the article, the report submission form and the 'report received' notification). A recent survey of authors and reviewers of Nature Research journals about attitudes towards transparency and openness in peer review, which had 2,742 respondents, showed clear support within the life sciences research community for the publication of anonymous referee reports. Given the ever-increasing numbers of research articles and the accompanying difficulties in finding referees, the survey was aimed at understanding the impact that moving to a more transparent model of peer review would have on agreement to review: $81 \%$ of respondents with a life sciences background said they would surely or very likely continue to review ( $9 \%$ weren't sure and $10 \%$ would not). One of the concerns raised in the survey was that it would take more time to prepare a publication-ready report. In our view, however, the referee reports that are usually submitted for our papers are of high quality and publication ready, so no changes would be needed in that regard. Given the survey results and that transparent peer review is being adopted by an increasing number of journals, we hope not to have major problems in finding reviewers. Nevertheless, we have introduced a new option in our system to indicate when a reviewer declines to assess a paper owing to disagreement with the transparent review process and ask that you please let us know if this is the case.

The peer-review file will not be edited and will thus be a true reflection of the concerns raised about the work, how the authors tackled them and which ones may have been overruled by the editors. However, authors will be able to request that we redact information provided in the rebuttal to the referees that they may wish to subsequently publish elsewhere. We will also redact mentions of third-party content (providing a link instead) and, in the case of transferred manuscripts, referee reports obtained by journals that do not operate a transparent review process. The whole file will be chronologically ordered and will have its own subheading in the online article navigation menu to increase its visibility.

What do we aim to achieve? The most important aspect of this change is enhancing transparency and accountability in decisionmaking, but feedback from the community suggests that peer-review files are also valuable learning tools as useful examples of how to assess a paper, and that early career researchers use them in journal clubs when discussing articles, as referee reports provide alternative viewpoints and analysis. Perhaps not surprisingly, the peer-review files of unexpected or controversial findings tend to be more accessed.

We are also taking steps to increase the openness of the data in the studies that we publish. Authors are now informed, at the revision and acceptance in principle stages, of our strong support for public data availability and asked to deposit all data associated with the paper in a public data repository, or present it as supplementary information. Our preferred way to share large datasets is through public repositories, a list of which - curated by our colleagues at Scientific Data - can be found on the journal's website (https://go.nature. $\mathrm{com} / 34 \mathrm{OQshH}$ ). Some of these repositories can host data confidentially and provide anonymous access to peer reviewers before public release. They then coordinate public release of the data with publication, although it remains the author's responsibility to liaise with the repository to ensure this occurs on the appropriate date. The numerical data underlying a study's figures should be provided in Excel format as source data and will be linked to the relevant figures online. Deposition of some types of data will continue to be mandatory (https://go.nature. $\mathrm{com} / 2$ Yihsnm), as will be providing full, unprocessed gels as source data.

A description of where all the data can be found, be it repositories, supplementary information or source data, should be included in the data availability statement (which already includes all relevant accession codes) so that they are easy to find. If there are data that can only be shared on request, we will ask authors to explain why in communication with the editor and include this explanation in the data availability statement.

We would like to take this opportunity to reiterate our data and materials sharing policy (https://go.nature.com/2YAlrM2), whereby authors are required to make materials, data, code and associated protocols promptly available to readers without undue delays, unless restriction to access has been stated in the manuscript. After publication, readers who are met by refusal from the authors to comply with these policies should contact the Chief Editor of the journal. If we are unable to resolve a complaint, the journal may refer the matter to the authors' funding institution and/or publish a formal statement of correction stating that readers have been unable to obtain necessary materials to replicate the findings.

We are excited to take these steps to increase the transparency of our editorial process and the amount of data made available with our articles, and would very much welcome your feedback about these initiatives as we continue to evolve in the changing landscape of scientific publishing.

Published online: 19 December 2019 https://doi.org/10.1038/s41564-019-0652-x 